\title{
ELABORACIÓN DE UN MODELO NEURONAL ARTIFICIAL PARA LA ESTIMACIÓN DE LA DEMANDA BIOQUÍMICA DE OXÍGENO EN AGUAS MARINAS
}

\author{
DEVELOPMENT OF AN ARTIFICIAL NEURAL NETWORK \\ MODEL FOR ESTIMATION OF BOD IN SEAWATERS
}

\author{
${ }^{1}$ Reynaldo José Meza Castellar \\ ${ }^{2}$ Luis Octavio González Salcedo \\ ${ }^{1}$ Ingeniero Ambiental, Universidad Nacional de Colombia, Palmira, Colombia \\ ${ }^{2}$ Doctor en Ingeniería, Universidad Nacional de Colombia, Palmira, Colombia \\ ${ }^{1}$ rjmezac@unal.edu.co; ${ }^{2}$ logonzalezsa@unal.edu.co
}

\section{RESUMEN}

Contextualización: los modelos neuronales artificiales son modelos diseñados a partir de métodos numéricos denominados Redes Neuronales Artificiales. El uso de estos, como herramienta de predicción de la Demanda Bioquímica de Oxígeno (DBO), ha demostrado diversas ventajas, entre otras, la reducción del tiempo y los costos económicos asociados a este parámetro. La DBO generalmente requiere de 5 a 7 días, así como múltiples reactivos químicos, para poder establecer los niveles de materiales orgánicos en las aguas.

Vacío de investigación: los modelos neuronales artificiales permiten calcular la DBO en tiempo real, a partir de variables fisicoquímicas registradas in situ. A pesar de ello, las redes neuronales artificiales no han sido utilizadas hasta ahora como método de estimación de la DBO en aguas marinas de Colombia.
Propósito del estudio: teniendo en cuenta este aspecto, en la presente investigación se elaboró un modelo neuronal artificial que permite estimar la DBPO en aguas del Mar Caribe Colombiano.

Metodología: para elaborar el modelo fue necesario realizar cinco simulaciones (constituidas por un número de 2 a 3 capas ocultas, y de 5 a 20 neuronas por capa). El desempeño predictivo de cada una de estas se evaluó a través del coeficiente de correlación.

Resultados y conclusiones: los valores más altos de este indicador estadístico $(0,937,0,951$, 0,953, y 0,941), se obtuvieron para el modelo que utilizó 3 capas, de 20 neuronas cada una, en sus cuatro etapas de aprendizaje (entrenamiento, validación, prueba, y todos los datos). Estas cifras indican un cercano ajuste entre los datos 
observados y las estimaciones hechas por la red. Estos resultados demuestran además que la Demanda Bioquímica de Oxígeno puede ser estimada numéricamente, en aguas marinas, a través de modelos neuronales artificiales.

Palabras clave: Levenberg-Marquardt; Mar Caribe; neurona.

\section{ABSTRACT}

Contextualization: Artificial Neural Networks are models designed from numerical methods called Artificial Neural Networks. The use of these, as a Biochemical Oxygen Demand (BOD) prediction tool, has shown various advantages, among others, the reduction of time and the economic costs associated with this parameter. BOD usually requires 5 to 7 days, as well as multiple chemical reagents, to obtain the levels of organic materials in the waters.

knowledge gap: Artificial Neural Networks models allow calculating BOD in real time from physicochemical variables recorded in situ. Despite this, artificial neural networks have not been used until now as a method of estimating $\mathrm{BOD}$ in Colombian seawaters.
Purpose: Taking this aspect into account, an artificial neural network model that allows estimating the BOD in waters of the Colombian Caribbean Sea was developed in this research.

Methodology: For the elaboration of the model it was necessary to carry out five simulations (consisting of a number of 2 to 3 hidden layers, and 5 to 20 neurons per layer). The predictive performance of each of these simulations was evaluated through the correlation coefficient.

Results and conclusions: The highest values of this statistical indicator $(0.937,0.951$, 0.953, and 0.941), were obtained for the model that used 3 layers, of 20 neurons each, in its four learning sets (training, validation, testing, and all data). These values indicate a close fit between the observed data and estimations made by the network. These results also demonstrate that Biochemical Oxygen Demand can be estimated numerically, in seawaters, through artificial neural networks models.

Keywords: Levenberg-Marquardt; Caribbean Sea; neuron.

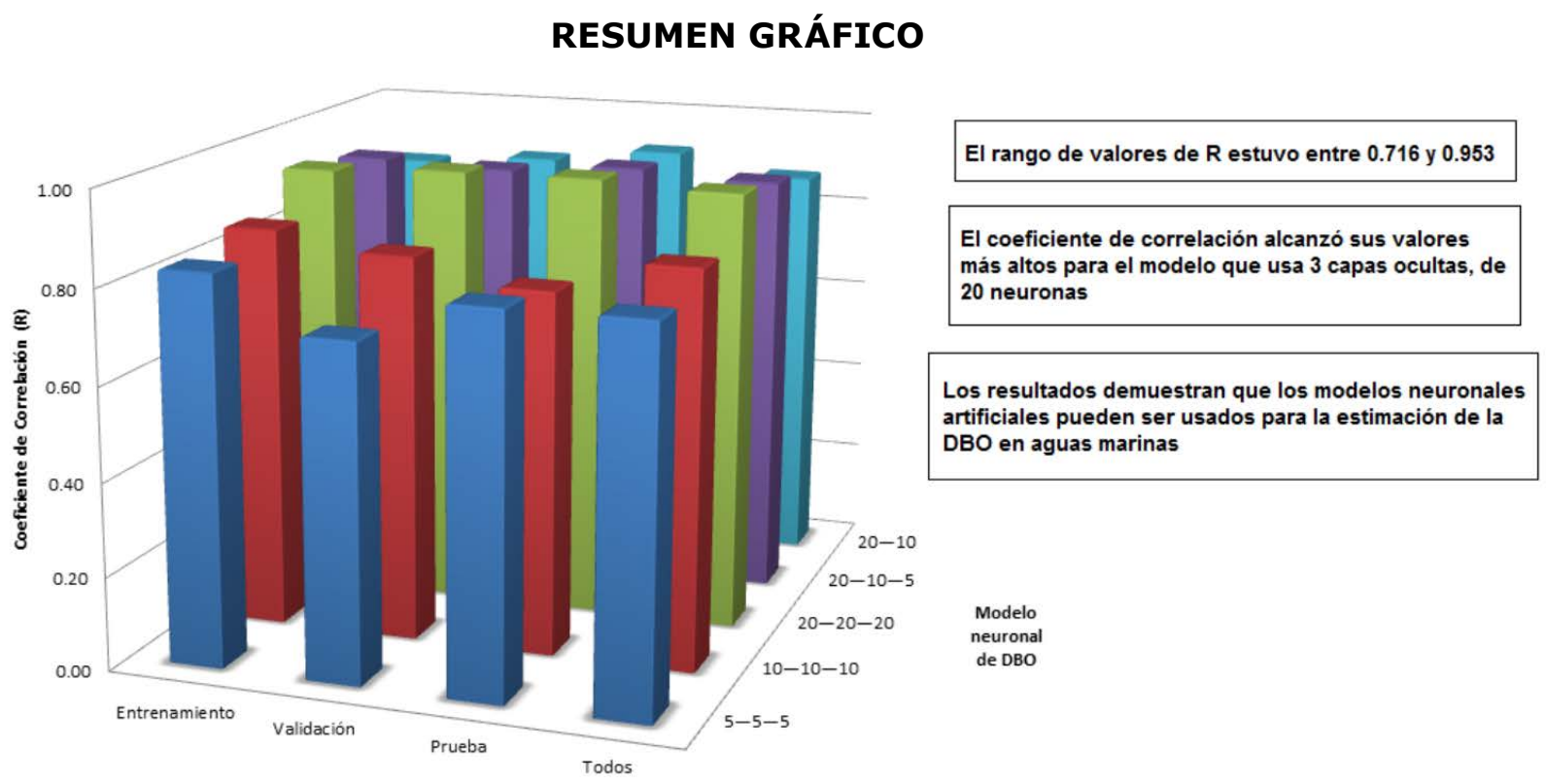




\section{INTRODUCCIÓN}

La demanda bioquímica de oxígeno (DBO) es un parámetro que representa la cantidad de oxígeno disuelto en el agua que es consumido o "demandado" por microorganismos durante la degradación de compuestos orgánicos (APHA, 2005). Esta, es también una medida indirecta del contenido de sustancias orgánicas biodegradables presentes en una muestra de agua (Matos et al., 2014). La estimación experimental de este parámetro generalmente requiere un periodo de 5 a 7 días ( $y$ en algunos casos, de 20 a 25 días), el uso de múltiples reactivos químicos, y el empleo de personal capacitado en el manejo de estas sustancias (APHA, 2005; Simon et al., 2011; Tachibana et al., 2013). Con el fin de reducir el tiempo y los costos económicos que este procedimiento involucra, en la última década, se han propuesto diversos métodos numéricos como alternativas de estimación de la DBO (Abyaneh, 2014). Dentro de estos, se han usado, para tal fin, estimaciones usando Redes Neuronales Artificiales (RNA), de las cuales se han obtenido resultados de predicción mejores a los obtenidos con otros modelos matemáticos convencionales y de regresión estadística (Tomenko, Ahmed, \& Popov, 2007 Dogan et al., 2014), los cuales se han aplicado para aguas continentales, siendo aún objeto de exploración, la estimación del parámetro usando las herramientas basadas en redes neuronales para aguas marinas (Meza, 2019). Considerando los anteriores aspectos, en este estudio, se propuso la elaboración de un modelo neuronal para la estimación de la DBO en aguas marinas del Departamento de Bolívar (Colombia). Para comprender la forma en que fue elaborado este modelo, a continuación, se describen algunos de los conceptos más relevantes.

\section{Demanda bioquímica de oxígeno}

La DBO es un proceso de tipo biológico y químico en el cual los materiales orgánicos constituidos por carbono $\left(\mathrm{C}_{\mathrm{x}} \mathrm{H}_{\mathrm{y}} \mathrm{O}_{\mathrm{z}}\right)$, y los compuestos nitrogenados provenientes de la hidrólisis de las proteínas $\left(\mathrm{NH}, \mathrm{NO}^{-}\right)$se oxidan en presencia de microorganismos (Phu, 2014):

$$
\mathrm{C}_{x} \mathrm{H}_{y} \mathrm{O}_{z}+\mathrm{O}_{2}+\underset{(\text { Ecuación 1) }}{\text { microorganismos }} \rightarrow \mathrm{CO}_{2}+\mathrm{H}_{2} \mathrm{O}
$$

$2 \mathrm{NH}_{3}+3 \mathrm{O}_{2}+$ bacterias $\rightarrow 2 \mathrm{NO}_{2}^{-}+2 \mathrm{H}^{+}+2 \mathrm{H}_{2} \mathrm{O}$ (Ecuación 2)

\section{$\mathbf{2} \mathrm{NO}_{2}^{-}+\mathrm{O}_{2}+$ bacterias $\rightarrow \mathbf{2} \mathrm{NO}_{3}^{-}$}

(Ecuación 3)

El oxígeno consumido en estas reacciones (generalmente expresado en $\mathrm{mg} / \mathrm{L}$ ) puede ser cuantificado experimentalmente a través de un procedimiento en el que se replican las condiciones ambientales del proceso. La guía Standard Methods for the Examination of Water and Wastewater, en su sección 5210, describe el protocolo utilizado comúnmente para estimar la DBO en laboratorio (APHA, 2005). Debido a que este procedimiento se fundamenta en las reacciones de consumo de oxígeno para aguas continentales, diversos investigadores consideran que este puede ser inadecuado para la determinación de la DBO en aguas marinas o bajo condiciones extremas de temperatura (Howington et al., 1994; Simon et al., 2011; Matos et al., 2014).

En los mares, la demanda bioquímica de oxígeno se origina por la descomposición de los compuestos orgánicos derivados de la fotosíntesis del fitoplancton, así como en la oxidación de la materia orgánica proveniente de los ríos y áreas urbanas (Tuchkovenko \& Lonin, 2003; INVEMAR, 2016). La DBO en las aguas marinas puede atribuirse también a una disminución del oxígeno producido por microalgas, o a una reducción del $\mathrm{O} 2$ soluble procedente del aire (Naimpally y Rosselot, 2014). Normalmente, en áreas marinas naturales, como los 
arrecifes de coral y bosques de manglar, la DBO no suele sobrepasar los $2 \mathrm{mg} / \mathrm{L}$ (Simon et al., 2011; Gandaseca et al., 2016; Severiche et al., 2017). Sin embargo, es posible encontrar demandas mucho más altas (>11.800 mg/L), en zonas costeras contaminadas por aguas residuales, o destinadas a la acuacultura y a la actividad pesquera (Muthukumaran \& Baskaran, 2013; CARDIQUE, 2017).

En Colombia, se estima que anualmente las aguas marinas reciben cerca de 34.633 toneladas de materia orgánica biodegradable (en forma de DBO), desde los municipios costeros, y alrededor de 971.856 toneladas desde sus sistemas fluviales más importantes (INVEMAR, 2016). De estos últimos, el Mar Caribe recibe aproximadamente unas 954.465 toneladas anuales, es decir, un $98 \%$ de las cargas totales de la nación (INVEMAR, 2016). Debido a ello, la costa Caribe colombiana es una de las zonas que mayor atención requiere en relación al manejo de este tipo de sustancias.

\section{Redes neuronales artificiales}

Las redes neuronales artificiales, son técnicas Las redes neuronales artificiales, son técnicas matemáticas que imitan la estructura y comportamiento de las redes de neuronas biológicas (Barthakur et al., 2012). Generalmente, una red de neuronas artificiales se encuentra conformada por tres estructuras o niveles de procesamiento de información denominados "capas". En el primer nivel, la "capa de entrada", se reciben los datos de las distintas variables independientes del modelo. En el segundo nivel o "capa oculta", se procesa y transfiere la información proveniente de la capa de entrada a las capas adyacentes. En el último nivel, la "capa de salida", se generan los resultados del modelo (Singh et al., 2009; Araghinejad, 2014). Cada una de estas capas se encuentra constituida por un conjunto de subestructuras denominadas neuronas (Singh et al., 2009). Dichas neuronas utilizan un número real para la ponderación de los valores de entrada denominado "peso", así como una función matemática para la normalización de los datos llamada "función de transferencia" (Palani et al., 2008).

Para encontrar el patrón que más se ajusta a la respuesta deseada, una red neuronal artificial lleva a cabo un proceso denominado aprendizaje o entrenamiento. En él, la red identifica una combinación óptima de pesos que asegura que las salidas del modelo sean cercanas a las observaciones (Palani et al., 2008). Los datos del problema a resolver, que son usados durante esta fase se denominan conjunto de entrenamiento. En la práctica, junto con este, suelen usarse dos conjuntos de datos denominados conjunto de prueba y conjunto de validación, los cuales son utilizados para medir el desempeño de la red y garantizar que no hayan sobreajustes en las estimaciones (Raoufy et al., 2011).

Como parte de su entrenamiento, las RNA requieren además que el número de sus capas ocultas, y la cantidad de neuronas presentes en ellas, se modifiquen hasta obtener una combinación óptima de estas. Debido a que en el campo de la modelación neuronal no existen reglas estrictas para establecer el número adecuado de capas y neuronas en una red, usualmente, este proceso es realizado por medio de ensayo y error, o según el criterio del investigador (Tomenko et al., 2007).

En la práctica, el uso de las RNA ha demostrado ser una herramienta útil de estimación de la DBO y otros parámetros de calidad del agua (Abyaneh, 2014; Emamgholizadeh et al., 2014). Es por ello que este estudio tiene como principales objetivos: (a) elaborar cinco modelos neuronales de DBO con distinto número de neuronas; (b) calcular sus respectivos 
desempeños predictivos por medio de un indicador estadístico; y (c) establecer el modelo más adecuado a través de la comparación de los valores de este indicador.

\section{MATERIALES Y MÉTODOS}

\section{Área de estudio}

El estudio se llevó a cabo en aguas marinas del Departamento de Bolívar (Colombia). La recolección de las muestras de agua fue realizada por la Corporación Autónoma Regional del Canal del Dique (CARDIQUE), en 32 estaciones de monitoreo. Estas estaciones se encuentran localizadas entre el corregimiento de Galerazamba, al norte de Bolívar $\left(10^{\circ} 47^{\prime} 20.19^{\prime \prime} \mathrm{N}\right.$ $\left.75^{\circ} 15^{\prime} 50.74^{\prime \prime} \mathrm{W}\right)$, y la desembocadura del Caño Correa, al sur de la costa de este departamento $\left(10^{\circ} 3^{\prime} 28.52^{\prime \prime} \mathrm{N} 75^{\circ} 27^{\prime} 27.03^{\prime \prime} \mathrm{W}\right)$. - La ubicación de las estaciones de calidad del agua puede consultarse en CARDIQUE (2017) e INVEMAR (2017)-.

\section{Estimación experimental de las variables}

Los parámetros fisicoquímicos usados en esta investigación (sólidos suspendidos totales, salinidad, $\mathrm{pH}$, oxígeno disuelto, y DBO) fueron registrados por CARDIQUE, entre los años 2011 y 2014. La estimación experimental de éstos se hizo de acuerdo a la Edición 22 de la guía Standard Methods for the Examination of Water and Wastewater siguiendo el protocolo 4500-O-G método del electrodo de membrana para el oxígeno disuelto; el protocolo 4500-H-B Método electrométrico para el potencial de hidrógeno; el protocolo 2520-B Método de conductividad eléctrica para la salinidad; el protocolo 2540-D para los sólidos suspendidos totales; y los protocolos 5210-B Prueba de DBO 5-días y 4500-O-G, para la DBO (APHA, 2005). Un total de 345 registros de cada parámetro fueron utilizados para la elaboración del modelo.

\section{Elaboración del modelo neuronal de DBO.}

El modelo neuronal para la predicción de la demanda bioquímica de oxígeno, se elaboró a partir de una red tipo Perceptrón Multicapa (PMC) (ver Anexo y Araghinejad (2014)). En este, se usó la DBO como variable de salida, mientras que el oxígeno disuelto (OD), el potencial de hidrógeno $(\mathrm{pH})$, la salinidad (Sal), los sólidos suspendidos totales (SST), y las coordenadas geográficas de cada estación de monitoreo (Lat-Long), se usaron como entradas (Figura 1). La selección de estos parámetros se hizo con base en su influencia sobre el proceso de demanda bioquímica de oxígeno y teniendo en cuenta la frecuencia con que han sido usados en estudios previos (Šiljić et al., 2016; Meza, 2019).

Por su parte, el código para el entrenamiento del PMC se elaboró a partir del modelo desarrollado por González et al. (2012). En este, el PMC es entrenado por medio del número de capas ocultas y el número de neuronas ocultas. Además, requiere definir un algoritmo para el entrenamiento de la red (que permite acelerar el proceso de aprendizaje del PMC), un número de épocas (la cantidad de veces que el algoritmo de entrenamiento es aplicado al conjunto de datos), un indicador de desempeño (por medio del cual la red ajusta las predicciones a las observaciones), y un método para la partición del conjunto de datos (división aleatoria, por bloques, intervalos, o índices). Este código se encuentra escrito en el lenguaje de programación " $M$ " y en él se hizo uso de las herramientas disponibles en el "Neural Network Toolbox" del software MATLAB $®$ (Beale et al., 2015; Meza, 2019).

En la Tabla 1 se presentan los 5 modelos (o arquitecturas) del perceptrón que fueron usados en la investigación. Estos modelos constan de entre 2 a 3 capas ocultas, y desde 5 hasta 20 
neuronas ocultas por capa. En cada modelo, el número de neuronas de la capa de entrada (6 neuronas), correspondió al número de variables usadas como entradas. Para su entrenamiento, se usó un número de 100 épocas y la suma de los errores cuadráticos (SSE) como indicador de desempeño. (La función de este último indicador estadístico es distinta a la del indicador usado para validar los resultados del modelo. Para ello véase el anexo al final del artículo).
Los modelos fueron evaluados por medio del algoritmo de Levenberg-Marquardt (ver anexo), el cual, según Zayani et al. (2008), Mustafa et al. (2012), Ebtehaj \& Bonakdari (2016), y Meza (2019), posee un desempeño predictivo superior al de otras metodologías de entrenamiento del PMC y, de acuerdo con Singh et al. (2009) y Meza (2019), es el algoritmo más adecuado para hacer estimaciones de la DBO.

Tabla 1. Modelos del Perceptrón multicapa usados en el estudio.

\begin{tabular}{l|c|c|c}
\hline \multirow{2}{*}{ Modelo } & \multicolumn{3}{c}{ Número de neuronas ocultas } \\
\cline { 2 - 4 } & Primera capa & Segunda capa & Tercera capa \\
\hline Modelo 1 & 5 & 5 & 5 \\
\hline Modelo 2 & 10 & 10 & 10 \\
\hline Modelo 3 & 20 & 20 & 20 \\
\hline Modelo 4 & 20 & 10 & 5 \\
\hline Modelo 5 & 20 & 10 & - \\
\hline
\end{tabular}

Fuente: Autores

Por su parte, el método usado para dividir el entrenamiento, validación, prueba, y todos los conjunto de datos fue de tipo aleatorio (dividerand). Este último divide el conjunto de obdatos) y asigna aleatoriamente a cada uno de servaciones en cuatro subconjuntos (Ilamados ellos un porcentaje del total de datos (70\%, $15 \%, 15 \%$, y $100 \%$, respectivamente).

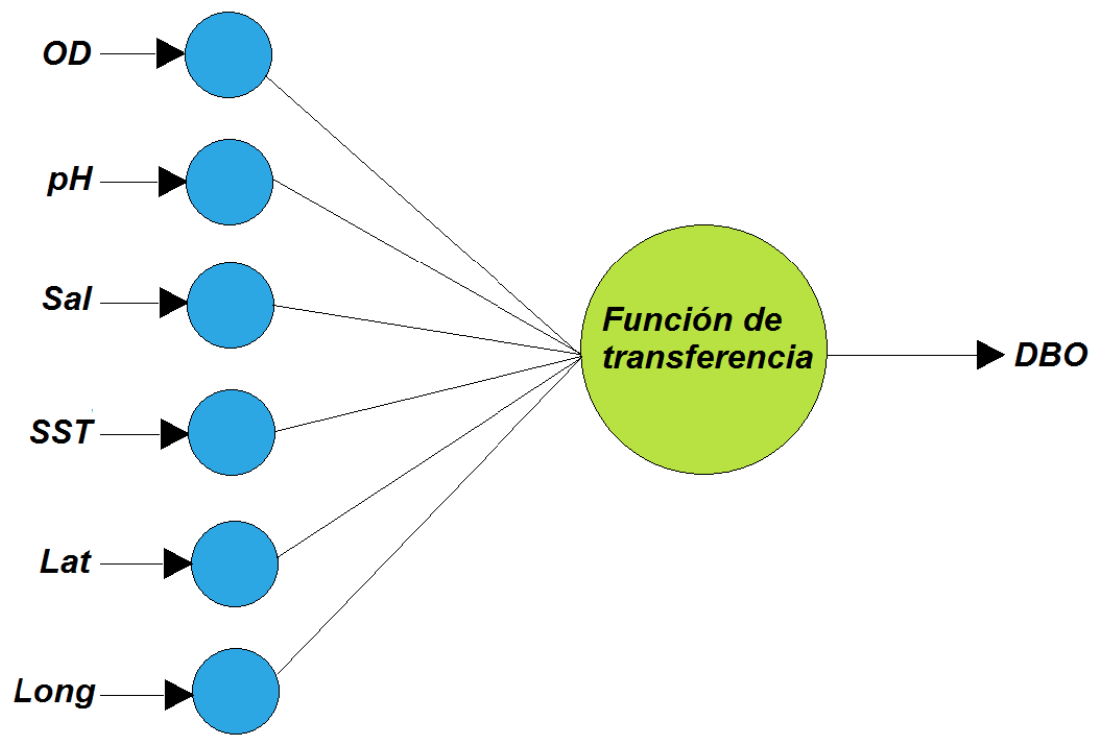

Figura 1. Representación conceptual de la neurona artificial utilizada en el estudio.

Fuente: Meza (2019). 


\section{ANÁLISIS ESTADÍSTICO}

Para poder comprobar la efectividad de cada modelo como método de estimación de la DBO, fue necesario aplicar un indicador estadístico al final de las distintas etapas del aprendizaje. Una de las pruebas estadísticas que se usan comúnmente en la evaluación del desempeño de las redes neuronales artificiales es el coeficiente de correlación $(R)$ :

$$
R=\frac{\sum_{i=1}^{n} \sum\left(X_{i}-\bar{X}\right)\left(Y_{i}-\bar{Y}\right)}{\sqrt{\sum_{i=1}^{n} \sum\left(X_{i}-\bar{X}\right)^{2}\left(Y_{i}-\bar{Y}\right)^{2}}}
$$

(Ecuación 4)

En la ecuación anterior, $n$ es el número total de observaciones; $X_{\mathrm{i}}$ y $Y_{\mathrm{i}}$ son los valores observados y estimados, respectivamente. $\bar{X}$ e $\bar{Y}$ son los valores promedio de todas las observaciones $X$ y estimaciones $Y$ (Abyaneh, 2014). Un $\mathrm{R}$ igual a 1 , indica un ajuste perfecto entre los resultados del modelo y los valores de DBO observados. Mientras que un $\mathrm{R}$ igual a cero, $\mathrm{O}$ negativo describe una correlación nula entre la predicción y la observación de la DBO (Gulyani et al., 2015).

Este indicador de desempeño se encuentra incluido en los códigos del PMC y sus valores fueron calculados de manera automática luego del entrenamiento. Además, fue usado como criterio de comparación de las distintas arquitecturas de la red. El modelo con el cual se obtuvieron los valores más altos del coeficiente de correlación (calculados de manera simultánea para las cuatro fases del aprendizaje), fue elegido como el más adecuado para hacer estimaciones de la DBO.

\section{RESULTADOS Y DISCUSIÓN}

En la Tabla 2 puede verse que la red Perceptrón multicapa, entrenada con el algoritmo de Levenberg-Marquardt, presentó valores del coeficiente de correlación entre 0,716 y 0,953. Sus cifras más bajas de $\mathrm{R}$ fueron calculadas en el modelo 1 (0,830 entrenamiento, 0,716 validación, y 0,806 todos), y el modelo $2(0,775$ prueba), mientras que los valores más altos se calcularon para todas las etapas de aprendizaje del modelo $4(0,916,0,907,0,927$, y 0,914$)$, y el modelo 3 (0,937, 0,951, 0,953, y 0,941). Debido a que este último obtuvo los $\mathrm{R}$ más altos de todas las simulaciones (en todas sus fases de aprendizaje y simultáneamente), fue seleccionado como el modelo más adecuado para calcular la demanda bioquímica de oxígeno.

En la tabla puede verse también que los valores de $\mathrm{R}$ se incrementaron gradualmente con el aumento en el número de neuronas ocultas (lo cual indicaría una posible influencia de la complejidad de la red sobre el desempeño predictivo de la misma); sin embargo, este comportamiento no se observó durante la fase de prueba posiblemente por el uso de la división aleatoria del conjunto de datos (ÁguilaMartínez, 2017)

Pese a estos óptimos resultados, debe tenerse en cuenta que el número de neuronas y capas ocultas no guarda relación con la DBO pues, para realizar sus cálculos, los modelos neuronales no utilizan ecuaciones que describan el proceso de demanda bioquímica de oxígeno (Emamgholizadeh et al., 2014). En este tipo de modelos, las neuronas solo representan un segmento de la función matemática que la red construye a partir del conjunto de observaciones. 
Tabla 2. Valores de R para el algoritmo de Levenberg-Marquardt

\begin{tabular}{|c|c|c|c|c|c|}
\hline \multicolumn{6}{|c|}{ Algoritmo de Levenberg-Marquardt } \\
\hline Modelo & Arquitectura & R entrenamiento & R validación & R prueba & $\mathbf{R}$ todos \\
\hline 1 & $5-5-5$ & 0,830 & 0,716 & 0,805 & 0,806 \\
\hline 2 & $10-10-10$ & 0,861 & 0,825 & 0,775 & 0,845 \\
\hline 3 & $20-20-20$ & 0,937 & 0,951 & 0,953 & 0,941 \\
\hline 4 & $20-10-5$ & 0,916 & 0,907 & 0,927 & 0,914 \\
\hline 5 & $20-10$ & 0,863 & 0,884 & 0,916 & 0,874 \\
\hline
\end{tabular}

Fuente: Autores

\section{CONCLUSIONES}

Se demostró que los modelos elaborados con redes neuronales artificiales se pueden usar para la estimación de la DBO en aguas marinas. La red neuronal utilizada en el diseño de estos modelos (el Perceptrón multicapa), alcanzó un alto desempeño al ser entrenada con el algoritmo de Levenberg-Marquardt ( $\mathrm{R}>$ $0,9)$. Al utilizar este último, en conjunto con 60 neuronas y tres capas ocultas, se obtuvieron los desempeños más altos del estudio.

A fin de dar mayor validez a los resultados obtenidos en este estudio, se recomienda llevar a cabo investigaciones en otras regiones costeras de Colombia, así como utilizar el modelo en situaciones reales (en el cálculo de datos faltantes de DBO, por ejemplo). También se sugiere que se utilicen bases de datos de mayor tamaño en el desarrollo de nuevos modelos, pues esto permite mejorar el desempeño predictivo de las RNA.

En la actualidad, las redes neuronales artificiales poseen un amplio campo de investigación y desarrollo. Este es otro motivo para seguir realizando estudios sobre modelación neuronal de la demanda bioquímica de oxígeno. A futuro se espera que se puedan usar los modelos neuronales de DBO en tiempo real, y que su uso llegue a ser una alternativa a los métodos convencionales de estimación de la DBO.

\section{CONTRIBUCIÓN DE LA AUTORÍA}

Primer autor: Metodología, investigación, análisis de datos, escritura-borrador original. Segundo autor: Metodología, supervisión, análisis de datos, escritura-revisión y edición.

\section{AGRADECIMIENTOS}

A la Corporación Autónoma Regional del Canal del Dique-CARDIQUE por suministrar los datos para la realización de esta investigación. Este artículo se encuentra basado en los resultados de la tesis de maestría de uno de los autores.

\section{LITERATURA CITADA}

Abyaneh, H. (2014). Evaluation of multivariate linear regression and artificial neural networks in prediction of water quality parameters. Journal of Environmental Health Science \& Engineering, 12(40), 1-8. https://doi.org/10.1186/2052$336 \mathrm{X}-12-40$

Águila-Martínez, J. (2017). Aprendizaje supervisado en conjuntos de datos no balanceados con Redes Neuronales Artificiales. Métodos de mejora de rendimiento para modelos de clasificación binaria en diagnóstico médico. Universitat Rovira i Virgili.

American Public Health Association (APHA). (2005). Standard Methods for the Examination of Water and Wastewater (21st ed.). Washington, DC: American Public Health Association (APHA).

Araghinejad, S. (2014). Artificial Neural Networks.

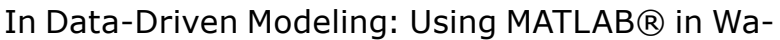
ter Resources and Environmental Engineering. 
Water Science and Technology Library, 67. https://doi.org/10.1007/978-94-007-7506-0

Barthakur, M., Thakuria, T., y Sarma, K. K. (2012). Artificial Neural Network (ANN) Based Object Recognition Using Multiple Feature Sets. Soft Computing Techniques in Vision Science, (395), 127-135. https://doi.org/10.1007/978-3-642-25507-6_11

Beale, M. H., Hagan, M. T., y Demuth, H. B. (2015). Neural Network Toolbox User's Guide. MathWorks, (R2015b), pp. 1-410.

Corporación Autónoma Regional del Canal del Dique (CARDIQUE). (2017). Evaluación de la calidad de las aguas en la Ciénaga de la Virgen. Corporación Autónoma Regional del Canal del Dique-CARDIQUE.

Dogan, E., Ates, A., Yilmaz, E. C., y Eren, B. (2014). Application of Artificial Neural Networks to Estimate Wastewater Treatment Plant Inlet Biochemical Oxygen Demand. Environmental Science \& Technology, 33(2), 482-489.

Ebtehaj, I., \& Bonakdari, H. (2016). Bed load sediment transport estimation in a clean pipe using multilayer perceptron with different training algorithms. KSCE Journal of Civil Engineering, 20(2), pp. 581-589. https://doi.org/10.1007/ s12205-015-0630-7

Emamgholizadeh, S., Kashi, H., Marofpoor, I., y Zalaghi, E. (2014). Prediction of water quality parameters of Karoon River (Iran) by artificial intelligence-based models. International Journal of Environmental Science and Technology, 11(3), 645-656. https://doi.org/10.1007/s13762-0130378-x

Gandaseca, S., Liyana, N., Wahab, A., Mustapha, A., y Pazi, M. (2016). Comparison of Water Quality Status of Disturbed and Undisturbed Mangrove Forest at Awat-Awat Lawas Sarawak. Open Journal of Forestry, 6, 14-18. https://doi. org/10.4236/ojf.2016.61002

González Salcedo, L. O., Guerrero Zúñiga, A. P., Delvasto Arjona, S., y Ernesto Will, A. L. (2012). Exploración con redes neuronales artificiales para estimar la resistencia a la compresión, en concretos fibroreforzados con acero. Ciencia e Ingeniería Neogranadina, 22(1), 19-41. https://doi.org/10.18359/rcin.247

Gulyani, B. B., Mangai, J. A., y Fathima, A. (2015). An Approach for Predicting River Water Quality Using Data Mining Technique. Lecture Notes in Computer Science (Including Subseries
Lecture Notes in Artificial Intelligence and Lecture Notes in Bioinformatics), 9165, pp. 233-243. https://doi.org/10.1007/978-3-319-20910-4_17

Howington, J. P., McFeters, G. A., Jones, W. L., y Smith, J. J. (1994). The effect of low temperature on BOD in Antarctic seawater. Water Resources, 28(12), 2585-2587. https://doi.org/10.1016/0043-1354(94)90078-7

Instituto de Investigaciones Marinas y Costeras (INVEMAR). (2016). Informe del estado de los ambientes y recursos marinos y costeros de Colombia. Año 2015.

Instituto de Investigaciones Marinas y Costeras (INVEMAR). (2017). Diagnóstico y Evaluación de la Calidad de las Aguas Marinas y Costeras en el Caribe y Pacífico Colombianos. Informe Técnico 2016.

Matos, M. P. de, Borges, A. C., Matos, A. T. de, Silva, E. F. da y Martinez, M. A. (2014). Effect of time-temperature binomial in obtaining biochemical oxygen demand of different wastewaters. Engenharia Agrícola, 34(2), 332-340. https://doi. org/10.1590/S0100-69162014000200014

Meza, R. (2019). Elaboración y uso de un modelo neuronal para la estimación de la DBO5. Caso de estudio: Costa Caribe del Departamento de Bolívar (Tesis de Maestría). Universidad Nacional de Colombia, Colombia.

Mustafa, M. R., Rezaur, R. B., Saiedi, S., y Isa, M. H. (2012). River suspended sediment prediction using various multilayer perceptron neural network training algorithms-A case study in Malaysia. Water Resources Management, 26(7), 18791897. https://doi.org/10.1007/s11269-012-9992-5

Muthukumaran, S., y Baskaran, K. (2013). Organic and nutrient reduction in a fish processing facility - A case study. International Biodeterioration and Biodegradation, 85, 563-570. https://doi.org/10.1016/j.ibiod.2013.03.023

Naimpally, A., y Rosselot, K. S. (2014). Water and Wastewater. In Environmental engineering: Review for the professional engineering examination. pp. 1-61. https://doi.org/10.1007/978-0-387-49930-7_1

Palani, S., Liong, S. Y., y Tkalich, P. (2008). An ANN application for water quality forecasting. Marine Pollution Bulletin, 56(9), 1586-1597. https://doi.org/10.1016/j.marpolbul.2008.05.021

Phu, S. T. P. (2014). Research on the Correlation Between Chlorophyll-a and Organic Matter BOD, COD, Phosphorus, and Total Nitrogen in 
Stagnant Lake Basins. In Sustainable Living with Environmental Risks, pp. 177-191. https://doi. org/10.1007/978-4-431-54804-1_15

Raoufy, M. R., Eftekhari, P., Gharibzadeh, S., y Masjedi, M. R. (2011). Predicting arterial blood gas values from venous samples in patients with acute exacerbation chronic obstructive pulmonary disease using artificial neural network. Journal of Medical Systems, 35(4), 483-488. https://doi.org/10.1007/s10916-009-9384-4

Severiche, C. A., Baldiris, I., Acosta, J. C., Bedoya, E. A., Castro, I., y Pacheco, H. (2017). Multivariate Analysis of Water Quality in Rosario Islands National Park (Colombia). American Journal of Engineering Research, 6(6), 136-144.

Šiljić, A., Antanasijević, D., Perić-Grujić, A., Ristić, M., y Pocajt, V. (2016). Artificial neural network modelling of biological oxygen demand in rivers at the national level with input selection based on Monte Carlo simulations. Environmental Science and Pollution Research, 23(4), 3978-3979. https://doi.org/10.1007/s11356-015-5978-1

Simon, F. X., Penru, Y., Guastalli, A. R., Llorens, J., \& Baig, S. (2011). Improvement of the analysis of the biochemical oxygen demand (BOD) of Mediterranean seawater by seeding control. Talanta, 85(1), 527-532. https://doi.org/10.1016/j.talanta.2011.04.032
Singh, K. P., Basant, A., Malik, A., \& Jain, G. (2009). Artificial neural network modeling of the river water quality-A case study. Ecological Modelling, 220, 888-895. https://doi.org/10.1016/j.ecolmodel.2009.01.004

Tachibana, K., Urano, Y., \& Numata, K. (2013). Biodegradability of nylon 4 film in a marine environment. Polymer Degradation and Stability Journal, 98, 1847-1851. https://doi.org/10.1016/j.polymdegradstab.2013.05.007

Tomenko, V., Ahmed, S., \& Popov, V. (2007). Modelling constructed wetland treatment system performance. Ecological Modelling, 205, 355-364. https://doi.org/10.1016/j.ecolmodel.2007.02.030

Tuchkovenko, Y. S., \& Lonin, S. A. (2003). Mathematical model of the oxygen regime of Cartagena Bay. Ecological Modelling, 165(1), 91-106. https:// doi.org/10.1016/S0304-3800(03)00064-4

Zayani, R., Bouallegue, R., \& Roviras, D. (2008). Adaptive predistortions based on neural networks associated with Levenberg-Marquardt algorithm for satellite down links. Eurasip Journal on Wireless Communications and Networking, 2008(132729), 1-15. https://doi. org/10.1155/2008/132729
Conflicto de Intereses Los autores declaran no tener ningún conflicto de intereses 